\title{
The Analysis of Korean Clinical Studies on the Effect of Pharmacopuncture for Whiplash Injury after Traffic Accidents
}

\author{
Jung Min Yun ${ }^{1}$, Na Yeon Hur ${ }^{1}$, Kyeong Han $\mathrm{Kim}^{2}$ * \\ ${ }^{1}$ College of Korean Medicine, Woosuk University, Jeonju, Republic of Korea \\ ${ }^{2}$ Department of Preventive Medicine, College of Korean Medicine, Woosuk University, Jeonju, Republic of Korea
}

Received November 4, 2020

Reviewed November 6, 2020

Accepted November 26, 2020
Objectives: Whiplash injury is one of the major diseases in recent times because of increasing traffic accidents. This review aims to analyze the overall trend of studies on pharmacopuncture for whiplash injury after traffic accidents.

Methods: We searched through 4 Korean electronic databases from 2001 up to October 2020 for relevant clinical studies for whiplash injury after traffic accidents, regardless of the patients' age, gender, or race. We included studies that had an intervention group receiving pharmacopuncture therapy with or without other additional treatments, and also included studies that had a control group receiving sham treatment or active treatment such as physical therapy and herbal medication. For the clinical outcomes, we did not place any restrictions on evaluation scales if they are objective metrics.

Results: We included 6 randomized controlled trials (RCTs) and 10 non-randomized controlled trials (nRCTs). $10 \mathrm{nRCTs}$ were divided into 4 categories that were case-control studies, case series, case report, and retrospective observational study. In RCTs, Hwangryunhaedoktang (黃蓮解毒湯) pharmacopuncture was the most frequently used. In nRCTs, Jungsong-ouhyul (中性疼血) pharmacopuncture, and bee venom pharmacopuncture were the most frequently used. As target points of Hwangryun-haedoktang pharmacopuncture, Ashi-points, Jianjing (GB21), and Fengchi (GB20) were the most frequently used. As target points of Jungsong-ouhyul pharmacopuncture, Ashi-points were the most frequently used. And as target points of Bee venom pharmacopuncture, Fengchi (GB20) was the most frequently used.

Conclusion: Hwangryun-haedoktang pharmacopuncture, bee venom pharmacopuncture, and Jungsong-ouhyul pharmacopuncture were mainly used for whiplash injury, and their usual target points were Jianjing (GB21), Fengchi (GB20), and Ashi-points. However, a high level of evidence should be conducted through studies with systematic methodology in the future.

Keywords: pharmacopuncture, whiplash injury, systematic review

\section{INTRODUCTION}

In modern society, traffic accidents are constantly increasing as the number of cars increases, and they are major factors in injury [1]. The number of patients who prefer traditional Korean medicine treatment for traffic accident injury treatment is increasing, and according to '2017 Medical Expense Statistical
Index', the number of automobile insurance claims for Korean medical institutions recorded 6.89 million cases, an increase of $8.34 \%$ from 6.36 million cases in the previous year $[2,3]$.

Whiplash Injury Associated Disorder (WAD) refers to head, neck and related systemic syndrome caused by skeletal injury, soft tissue damage, or internal bruising occurring when the head of an occupant is sharply overextended or over bent due to 
sudden acceleration or deceleration in various automobile accidents including front, rear or side collisions of a vehicle [4]. Recently, Korean medical institutions have been using traditional Korean medicine treatments such as acupuncture treatment [5], pharmacopuncture therapy [6], chuna treatment [7], and herbal medicine treatment [8] for WAD, and the results also have been reported to be positive.

Pharmacopuncture, as a traditional Korean medicine technology independently developed, combines acupuncture therapy and herbal medicine therapy [9]. It is widely used for various diseases such as musculoskeletal diseases and nervous system diseases as a medical treatment in which a medication extracted and purified from a specific herbal medicine is injected into reaction points on body surface or acupuncture points where therapeutic effect appears [10]. Various studies on traditional Korean medicine treatment with pharmacopuncture have been conducted such as experimental studies, observational studies, technical studies, and randomized controlled clinical trials (RCTs) [11, 12].

The most damaged body parts in traffic accident injuries were cervical and lumbar spine, and there were many minor injuries that did not require urgent surgical treatment [13]. With regard to neck pain after traffic accidents, there are some clinical studies that describe the effect of a specific pharmacopuncture such as The Comparative Study on the Effect of Pharmacopuncture Treatment and Chuna Treatment for Neck Pain Caused by Traffic Accidents [14], and A retrospective study on the effects of Shinbaro pharmacopuncture and Jungsongouhyul pharmacopuncture on whiplash injury by traffic accident [15].

However, review research on general pharmacopuncture therapy used for neck pain after traffic accidents are insufficient. Therefore, in this study, in order to grasp the overall trend of research on pharmacopuncture for whiplash injury after traffic accidents in traditional Korean medicine, Korean research on pharmacopuncture therapy from 2001 to 2020 were classified and reviewed by the types of studies and then, further reclassified and analyzed by type of pharmacopuncture used.

\section{MATERIALS AND METHODS}

\section{Search method for identifying the studies}

The search engine consists of 4 search databases that provide original texts and bibliography of Korean medical databases:
RISS (Research Information Sharing Service), KISS (Korean studies Information Service System), OASIS (Oriental Medicine Advanced Searching Integrated System), NDSL (National Digital Science Library).

In order to search studies related to pharmacopuncture therapy for whiplash injury, the keywords used for search were 'pharmacopuncture AND traffic accident', 'acupuncture AND traffic accident', 'pharmacopuncture AND whiplash injury', 'acupuncture AND whiplash injury' and 'whiplash injury'.

\section{Inclusion criteria for this review}

\section{1) Study type}

In this study, we selected studies using pharmacopuncture therapy as a treatment for whiplash injury after traffic accidents, published in Korean journals before the search date (October 2020). With the inclusion criteria that includes all types of clinical studies with patients, we included not only randomized controlled trials (RCT), but also cohort studies, case-control studies, case series, and case reports. However, non-clinical studies including literature and animal studies, publications that were not formatted as research paper, and studies published before 2,000 were excluded.

\section{2) Participant of study}

This study included all clinical studies with patients diagnosed with neck pain induced by whiplash injury, regardless of diagnostic criteria, sex, age, race, and period from the date of onset.

\section{3) Type of therapeutic intervention}

All clinical studies that performed pharmacopuncture therapy in the experimental group were included. Studies using combination treatments including pharmacopuncture were also included. Studies using only other types of therapeutic interventions except pharmacopuncture therapy were excluded.

Therapeutic intervention of control group was not particularly limited, including sham treatment and active treatments (such as acupuncture, herbal medicine, pharmacopuncture and physical therapy).

Studies that did not use traditional Korean medicine treatment were excluded. Pharmacopuncture therapy, injecting a therapeutic solution purified from herbal medicines into related acupuncture points or Ashi-points, etc., is a traditional Korean medicine treatment. Therefore, it is different from other 
acupuncture treatment such as electroacupuncture or general acupuncture treatment without injecting drugs. Acupuncture treatments that do not use drug-injection or inject drugs other than drug solution purified from herbal medicine were excluded from the analysis target. In addition, there were no restriction on the types of herbal drugs used in pharmacopuncture therapy.

\section{4) Scale of rating}

All categories of scales that can be expressed as objective numerical values for neck pain that occurred after traffic accidents were included.

\section{Data collection, extraction and assessment method}

\section{1) Selection of studies}

For data extraction, two researchers (JM and NY) independently reviewed the title and abstract retrieved from 4 databases, and extracted studies that used pharmacopuncture therapy related to traditional Korean medicine for the treatment of whiplash injury that occurred after traffic accidents. After the researchers independently confirmed whether studies met the criteria for inclusion, they cross-verified their work. Any disagreements over the selected studies were resolved by discussing the issues with a third author and reaching a consensus. Subsequently, based on the full text, the extracted studies were further filtered by whether the studies were appropriate for our study.

\section{2) Classification of extracted studies}

The extracted clinical studies were classified into nonrandomized controlled trials (nRCTs) and RCTs. Then, nRCTs were classified according to the study design. It was subdivided into case-control study, case-series study, and case report, etc.

After that, to comprehensively analyze acupuncture points used by pharmacopuncture therapy, all target clinical studies were analyzed according to their types of pharmacopuncture therapy and then the acupuncture points used for the respective type.

\section{RESULTS}

\section{Search results}

As a result of using each database, a total of 310 Korean studies were searched. Out of 201 extracted studies excluding duplicated studies, a total of 18 studies done before 2000 were also excluded. Among the 183 studies, the following studies are excluded according to our exclusion criteria: 137 studies which did not use traditional Korean medicine treatment, 17 studies whose subjects were patients without neck pain, 4 non-clinical studies, 8 studies that did not use pharmacopuncture therapy, and 1 study where the original text could not be found. Totally 16 studies were obtained. These 16 studies were classified and analyzed as RCTs and nRCTs (Fig. 1).

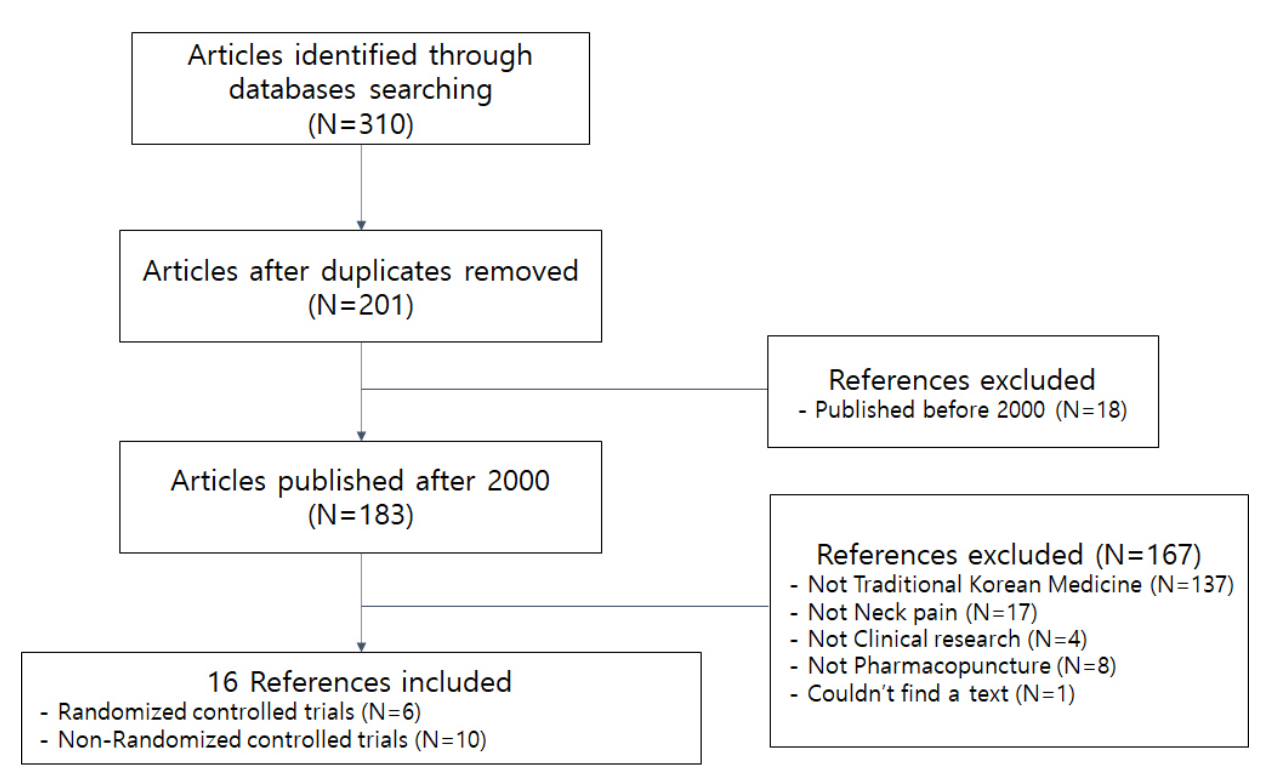

Figure 1. Flow chart. 


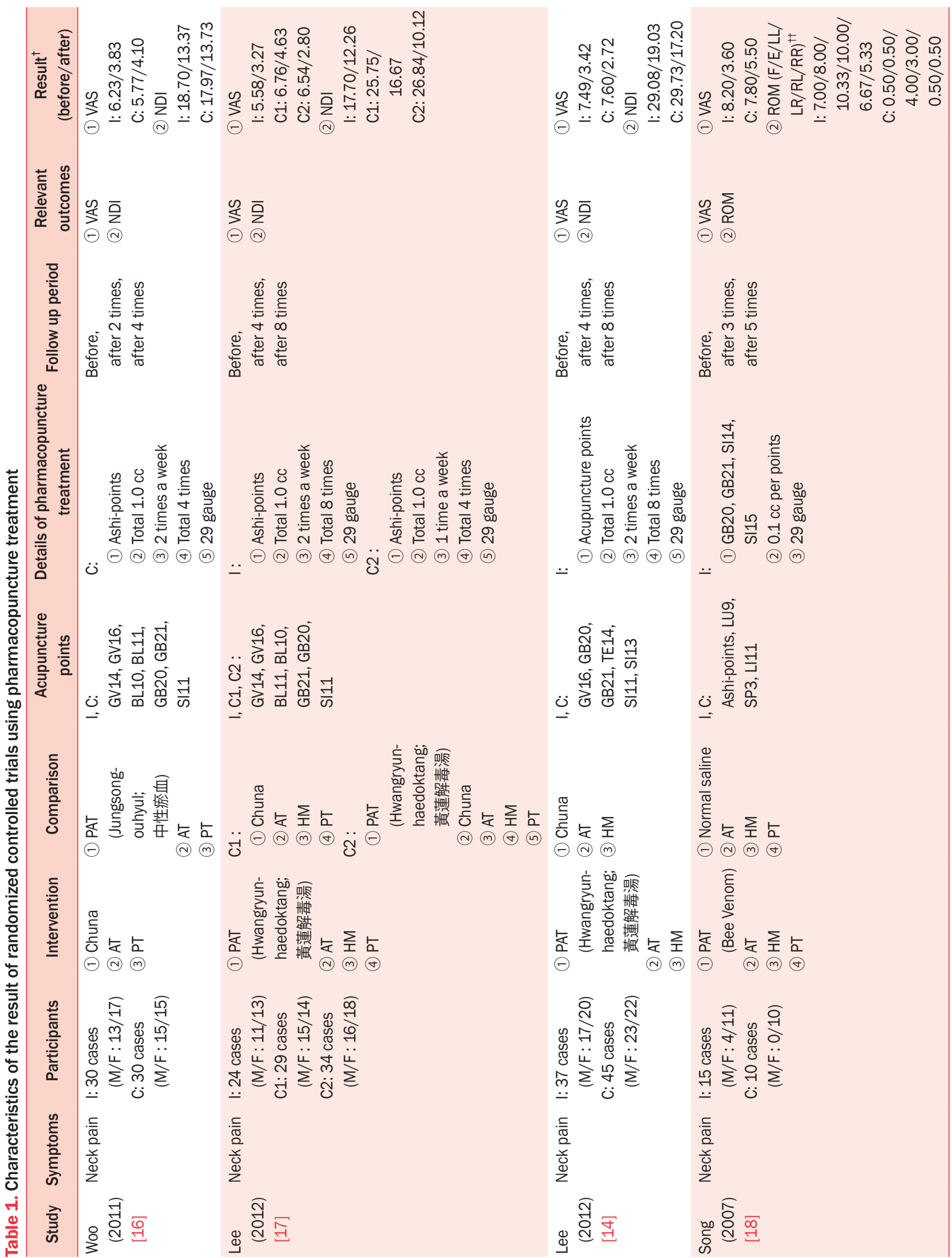




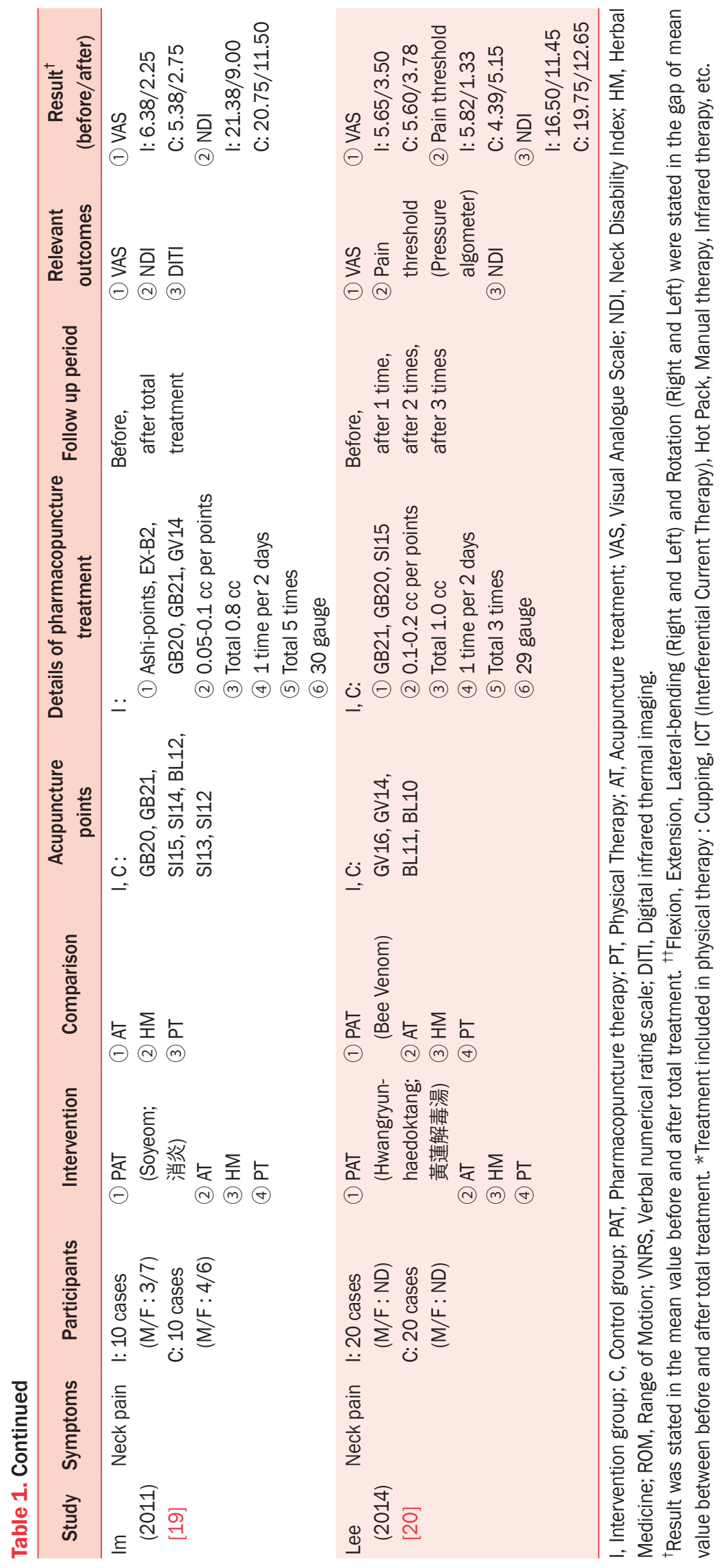




\section{Analysis of randomized controlled trials (RCTs)}

A total of 6 RCTs were analyzed by symptoms, study participants, intervention factors, acupuncture points, details of pharmacopuncture treatment, follow-up tests, and outcome values (Table 1).

\section{1) Intervention factors}

In the experimental group, the most frequently applied treatments along with pharmacopuncture were acupuncture treatment and herbal medicine, followed by physical therapy. There was no experimental group that used pharmacopuncture therapy alone, and when pharmacopuncture therapy was used as an intervention factor, all studies used combined treatments including pharmacopuncture therapy.

Acupuncture treatment was the most commonly used treatment in the control group, followed by herbal medicine treatment and physical treatment. And there were some cases that applied pharmacopuncture therapy, Chuna treatment, and physiological saline in the control group.

When acupuncture was used as an intervention factor, Jianjing (GB21), Fengchi (GB20), and Tianzong (SI11) were the most commonly used.

\section{2) Pharmacopuncture therapy}

The types of pharmacopuncture therapy used in the six studies were Jungsong-ouhyul (中性疼血) pharmacopuncture, Hwangryun-haedoktang (黃蓮解毒湯) pharmacopuncture, bee venom pharmacopuncture and Soyeom (消炎) pharmacopuncture. The most frequently used pharmacopuncture treatment was Hwangryun-haedoktang pharmacopuncture, followed by bee venom pharmacopuncture.

Hwangryun-haedoktang pharmacopuncture therapy was most often used in Jianjing (GB21) and Fengchi (GB20), and the method of injecting a total of $1.0 \mathrm{cc}$ using the $29 \mathrm{G}$ needle was the most applied. Other than those, Jianzhonshu (SI15), Jianwaishu (SI14), Ashi-points, Fengfu (GV16), Quyuan (SI13), Tianzong (SI11), and Jianliao (TE14) were used. And the most common treatment period of Hwangryun-haedoktang pharmacopuncture therapy was 2 times a week, with the total of 8 times.

Bee venom pharmacopuncture therapy was most often used at Jianjing (GB21), Fengchi (GB20), and Jianzhonshu (SI15), and the method of injecting $0.1 \mathrm{cc}$ into each point using the $29 \mathrm{G}$ needle was the most commonly applied. Their treatment period was different for each study.

Table 2. Classification of non-randomized controlled trials using pharmacopuncture treatment

\begin{tabular}{|c|c|c|c|}
\hline \multirow{2}{*}{ Study type } & \multicolumn{2}{|c|}{ Intervention } & \multirow{2}{*}{$\begin{array}{l}\text { Pharmacopuncture treatment contents } \\
\text { (acupuncture points) }\end{array}$} \\
\hline & Common & $\mathrm{I}-\mathrm{C}$ & \\
\hline \multirow[t]{5}{*}{ Case-control study } & AT, HM, PT, Chuna & I: PAT & I: Jungsong-ouhyul; 中性瘀血 (EX-B2, Ashi-points, GB20, GB21, GB14) \\
\hline & & C: PAT & C: Soyeom; 消炎 (EX-B2, Ashi-points, GB20, GB21, GB14) \\
\hline & PT & I: PAT & I: Bee Venom (GV15, GV16, GB12, GB20) \\
\hline & & C: AT & \\
\hline & AT, HM, PT & I: PAT & I: Jungsong-ouhyul; 中性瘀血 (EX-B2, Ashi-points) \\
\hline Case-series research & PAT, AT & & Bee Venom (BL10, GB20, GB21, SI11, SI13, SI14, SI15) \\
\hline Case report & PAT, AT, HM, PT, Chuna & & Hwangryun-haedoktang; 黃蓮解毒湯 (EX-B2, GB20, GB21, Ashi-points) \\
\hline \multirow[t]{10}{*}{ Retrospective observational study } & AT, HM & I: PAT & I: Shinbaro (Ashi-points) \\
\hline & & C: PAT & C: Jungsong-ouhyul; 中性瘀血 (Ashi-points) \\
\hline & AT, HM, PT & I: PAT & I: Muscle Relaxation (GB20, GB21, GV16, SI15) \\
\hline & & C: Chuna & \\
\hline & AT, HM, PT & I: PAT & I: Hwangryun-haedoktang; 黃蓮解毒湯 (Ashi-points) \\
\hline & & C1: PAT & C1: Bee Venom (Ashi-points) \\
\hline & & C2: PAT & C2: Jungsong-ouhyul; 中性瘀血 (Ashi-points) \\
\hline & AT, HM & I: PAT & I: Shinbaro (Ashi-points) \\
\hline & & C: PAT & C: Bee Venom (Ashi-points) \\
\hline & AT, HM, PT & I: PAT & I: Jungsong-ouhyul; 中性瘀血 (EX-B2, Ashi-points) \\
\hline
\end{tabular}

I, Intervention group; C, Control group; PAT, Pharmacopuncture therapy; AT, Acupuncture treatment; HM, Herbal Medicine; PT, Physical Therapy. 


\section{3) Result value}

Among the evaluation scales used, VAS (Visual Analogue Scale) and NDI (Neck Disability Index) were used in all 6 RCTs. As for the results, all studies showed positive results for neck pain after traffic accidents.

\section{Analysis of non-randomized controlled trials (nRCTs)}

A total of $10 \mathrm{nRCT}$ s were classified by study types to analyze the intervention factors (Table 2). There were 3 case-control studies, 1 case series, 1 case report, and 5 retrospective observational studies. The types of pharmacopuncture therapy used were Jungsong-ouhyul pharmacopuncture, Soyeom pharmacopuncture, Bee venom pharmacopuncture, Hwangryun-haedoktang pharmacopuncture, Shinbaro pharmacopuncture, and Muscle Relaxation pharmacopuncture. The most commonly used type was Jungsong-ouhyul pharmacopuncture, followed by bee venom pharmacopuncture.

Acupuncture treatment, herbal medicine, and physical therapy were the most common therapeutic interventions used with Jungsong-ouhyul pharmacopuncture therapy, and chuna therapy was applied in some cases. Physical therapy was the most commonly used treatment along with bee venom pharmacopuncture therapy, followed by acupuncture treatment.

\section{Acupuncture points used for each type of pharmacopuncture}

The 16 studies, including both RCTs and nRCTs, were classified by the types of pharmacopuncture therapy used, and the acupuncture points used for each pharmacopuncture were analyzed (Table 3).

Bee venom pharmacopuncture therapy used Fengchi (GB20) the most often, followed by Jianjing (GB21) and Jianzhonshu (SI15). Jungsong-ouhyul pharmacopuncture therapy used Ashipoints the most often. Hwangryun-haedoktang pharmacopuncture therapy used Ashi-points, Jianjing (GB21), and Fengchi (GB20) the most often. Shinbaro pharmacopuncture therapy used only Ashi-points. Muscle relaxation pharmacopuncture therapy used Jianjing (GB21), Fengchi (GB20), Fengfu (GV16), and Jianzhonshu (SI15). Soyeom pharmacopuncture therapy used Jiaji (EX-B2), Jianjing (GB21), Fengchi (GB20), and Dazhui (GV14).
Table 3. Classification by types of pharmacopuncture treatment

\begin{tabular}{|c|c|c|}
\hline $\begin{array}{c}\text { Type of } \\
\text { pharmacopuncture }\end{array}$ & \multicolumn{2}{|c|}{$\begin{array}{c}\text { Number of mainly used } \\
\text { acupuncture points }\end{array}$} \\
\hline \multirow[t]{11}{*}{ Bee Venom } & GB20 & 4 \\
\hline & GB21 & 3 \\
\hline & SI15 & 3 \\
\hline & Ashi-points & 2 \\
\hline & SI14 & 2 \\
\hline & GV15 & 1 \\
\hline & GV16 & 1 \\
\hline & GB12 & 1 \\
\hline & BL10 & 1 \\
\hline & SI11 & 1 \\
\hline & SI13 & 1 \\
\hline \multirow{5}{*}{$\begin{array}{l}\text { Jungsong-ouhyul; } \\
\text { 中性瘀血 }\end{array}$} & Ashi-points & 6 \\
\hline & EX-B2 & 2 \\
\hline & GB14 & 1 \\
\hline & GB20 & 1 \\
\hline & GB21 & 1 \\
\hline \multirow[t]{4}{*}{ Muscle relaxation } & GB20 & 1 \\
\hline & GB21 & 1 \\
\hline & GV16 & 1 \\
\hline & SI15 & 1 \\
\hline \multirow{9}{*}{$\begin{array}{l}\text { Hwangryun-Haedoktang; } \\
\text { 黃蓮解毒湯 }\end{array}$} & Ashi-points & 3 \\
\hline & GB20 & 3 \\
\hline & GB21 & 3 \\
\hline & EX-B2 & 1 \\
\hline & GV16 & 1 \\
\hline & TE14 & 1 \\
\hline & SI11 & 1 \\
\hline & SI13 & 1 \\
\hline & SI15 & 1 \\
\hline \multirow{5}{*}{$\begin{array}{l}\text { Soyeom; } \\
\text { 消炎 }\end{array}$} & Ashi-points & 1 \\
\hline & EX-B2 & 1 \\
\hline & GB20 & 1 \\
\hline & GB21 & 1 \\
\hline & GV14 & 1 \\
\hline Shinbaro & Ashi-points & 2 \\
\hline
\end{tabular}

\section{DISCUSSION}

In modern society, the use of automobiles continues to increase, and injuries caused by traffic accidents occupy a large part of social losses, so treatment and management of injuries 
are important factors not only medically but also socially [21]. Traffic accident patients are normal in diagnostic tests, but there are many cases where the after-effects persist for a long time even after treatment [22]. The cervical spine is one of the most common injured regions caused by traffic accidents, and this causes neck pain [13].

Unlike conventional acupuncture treatment, pharmacopuncture therapy has the advantage by injecting drugs to be absorbed directly into the tissues. Therefore, it can greatly reduce the amount of drug used and increase the therapeutic effect compared to oral drug of herbal medicine $[23,24]$. Furthermore, there is a report that this pharmacopuncture therapy was also used for patients with neck pain caused by traffic accidents and showed good therapeutic effects [25]. As of 2020, there have been many clinical studies on the treatment of traditional Korean medicine for patients with neck pain caused by traffic accidents, but there is a lack of systematic review studies on pharmacopuncture therapy related to whiplash injury. In this study, recognizing the necessity of systematic literature research on pharmacopuncture therapy for neck pain caused by traffic accidents, the trend of related research was investigated through Korean electronic databases.

Of the 16 studies that were the final analysis subjects, 6 were RCTs and 10 were nRCTs. As a result of analyzing six RCTs, Hwangryun-haedoktang pharmacopuncture therapy was the most commonly used, followed by bee venom pharmacopuncture. As a result of analyzing 10 studies that were nRCTs, Jungsong-ouhyul pharmacopuncture was used the most commonly used.

Hwangryun-haedoktang consists of Coptidis Rhizoma, Scutellariae Radix, Phellodendri Cortex, and Gardeniae Fructus. According to ${ }^{\circledR B a n g y a k h a p p y e o n}$ [26], it can decrease body temperature and detoxify body. Therefore, it can be used for symptoms such as chest tight, high fever, and thirsty. Hwangryun-haedoktang pharmacopuncture made by refining Hwangryun-haedoktang is also one of the Palgang-pharmacopunctures that have similar effects to those of Hwangryunhaedoktang [27], and it is mainly used to treat musculoskeletal diseases and skin diseases [28]. Usually, when a traffic accident occurs, various systemic symptoms such as pain, internal injuries, bleeding, and fever appear, so it is thought that Hwangryun-haedoktang pharmacopuncture therapy can be effectively used these symptoms. The most frequently used points in Hwangryun-haedoktang pharmacopuncture therapy are Ashipoints, Jianjing (GB21), and Fengchi (GB20), all of which are located on the cervical region.

Bee venom pharmacopuncture therapy is a treatment that cures diseases by extracting and processing bee venom and then injecting it into the lesion using a syringe [29]. It is known to activate the immune system and have anti-inflammatory, antibacterial, and anti-fungal effects. Also, it has been reported that it can be used for various musculoskeletal diseases [30]. However, Enzyme components including phospholipase A2 and hyaluronidase in bee venom could cause allergic symptoms. Recently, new pharmacopuncture called "sweet bee venom" was developed to reduce the risks of anaphylactic shock and it has been used in lots of medical institutions [18]. However, this study did not distinguish between bee venom pharmacopuncture and sweet bee venom pharmacopuncture, classifying them as the same type of pharmacopuncture therapy. It is thought that bee venom pharmacopuncture therapy can be effectively used for patients who have inflammatory symptoms due to traffic accidents, and can be used for the after-effects of traffic accidents in terms of activating the immune system even if inflammatory symptoms do not appear. The most frequently used points in bee venom pharmacopuncture therapy was Fengchi (GB20) on the cervical region.

Jungsong-ouhyul pharmacopuncture consists of Gardeniae Fructus, Corydalis Tuber, Olibanum, Myrrha, Persicae Semen, Paeoniae Radix, Salviae Miltiorrhizae Rrdix, and Sappan Lignum. In traditional Korean medicine, it can facilitate blood circulation, reduce pain, decrease body temperature, and do antiinflammatory function [27].

Since neck pain caused by traffic accidents is a symptom by Qi-massing (氣滯) and Blood-Massing (瘀血) in traditional Korean medicine, it can be treated with Jungsong-ouhyul pharmacopuncture's alleviation of fever and anti-inflammatory function [16]. The most frequently used points in Jungsong-ouhyul pharmacopuncture therapy were Ashi-points. Ashi points are where pain occurs when pressed and have the meaning of where Qi-massing or Blood-Massing has occurred.

Other than those, Soyeom pharmacopuncture, Shinbaro pharmacopuncture, and Muscle Relaxation pharmacopuncture were used as therapy for neck pain caused by traffic accidents. Soyeom pharmacopuncture is effective to reduce the musculoskeletal disorders such as ankle tendinitis, lumbar disc herniation $[19,31,32]$. It was reported that Shinbaro pharmacopuncture consists of herb having anti-inflammatory effect and nerve regeneration effect, and it was used to treat many musculoskeletal disorders such as lumbar disc herniation, lumbar lordosis 
$[33,34]$. Muscle relaxation pharmacopuncture was also reported to effectively treat neck pain caused by traffic accidents [35].

This study was able to confirm the trend of Korean research in the past 20 years of pharmacopuncture therapy studies, among the treatments for whiplash injury after traffic accidents. However, it has some limitations in that only Korean databases were used for search. Also, since we only used the search term 'pharmacopuncture' and 'acupuncture', this study could not include some terms that were called with the same meaning as pharmacopuncture, such as 'herb acupuncture', 'injection therapy'. And RCTs included in the final analysis have limitations in terms of qualitative evaluation as they do not properly report blinding methods or side effects. Among the points of the pharmacopuncture therapy used in each study, the location of Ashi-points varies from patient to patient depending on the area where the pain appears. Sometimes they can be the same area as other acupuncture points. Because it cannot be said that all patients got the same treatments in studies using Ashipoints, there has limitations in analysis. Finally, since none of these studies had a therapeutic intervention group that used only pharmacopuncture therapy, there may be limitations in accurately evaluating the effects of pharmacopuncture therapy.

Despite the limitations, our study is meaningful in that it has comprehensively summarized the pharmacopuncture therapy that can be used when neck pain occurs due to the after-effects of traffic accidents in the recent times when the number of traffic accidents is increasing.

Until now, many studies have been conducted on the effects, appropriate doses, and risks of pharmacopuncture therapy, but few studies have focused on summarizing the effective types of pharmacopuncture therapy as per disease. Therefore, even though pharmacopuncture therapy is used frequently in recent traditional Korean medicine treatment, the lack of those systematic studies is limiting its further application. Therefore, from now on, that kind of studies should be done more so that systematic treatments are possible.

\section{CONCLUSION}

The pharmacopuncture therapies used for whiplash injury were mainly Hwangryun-haedoktang pharmacopuncture, bee venom pharmacopuncture, and Jungsong-ouhyul pharmacopuncture. And their usual points were Jianjing (GB21), Fengchi (GB20), and Ashi-points. However, since several clinical studies, including RCTs, have various limitations in terms of research methodology, studies with a higher level of evidence should be conducted with more systematic methodology in the future.

\section{AUTHORS' CONTRIBUTIONS}

JM and NY contributed to the design of the study, reviewed, and summarized the articles, and analyzed the records. The study has been read and approved by all authors.

\section{CONFLICT OF INTEREST}

The authors declare that there are no conflicts of interest.

\section{ORCID}

Jung Min Yun, https://orcid.org/0000-0001-9101-6945

Na Yeon Hur, https://orcid.org/0000-0003-3378-7121

Kyeong Han Kim, https://orcid.org/0000-0003-4868-9145

\section{REFERENCES}

1. Cho SW. Survey on expectation of Korean medicine treatment in 39 cases by traffic accident. J Korean Med Rehabil. 2013;23(3): 187-99.

2. Park SY, Lee YK, Kim JS, Lim SC, Lee BH, Jung TY, et al. Survey of oriental medical care for traffic accident patients with automobile insurance; 544 cases report. J Korean Acupunct Moxib Soc. 2009;26(3):1-10.

3. Health Insurance Review \& Assessment Service. 2017 Medical expenses statistics. Wonju: Health Insurance Review \& Assessment Service; 2018. p. 135-7.

4. Korean Society of Chuna Manual Medicine for Spine and Nerves. Chuna manual medicine. 2nd ed. Seoul: Korean Society of Chuna Manual Medicine for Spine and Nerves; 2014. p. 36377.

5. Jeon JY, Yoo DJ, Maeng TH, Shim JW, Cho WY, Kim HS, et al. A review of clinical studies about acupuncture therapy for whiplash associated disorder. J Korean Med Rehabil. 2015;25(4):4754.

6. Kim YJ, Kim TR, Woo CH, Shin BC. Comparative effectiveness of Hwangryunhaedok-tang pharmacopuncture, essential bee venom pharmacopuncture and Jungsongouhyul pharmacopuncture for cervical pain caused by traffic accidents: a retrospective observational study. J Korean Med Rehabil. 2018;28(2):83-9.

7. Jeon YT, Park SH, Han KI, Lee JH, Ko YS. Trends of Chuna manual therapy on traffic accident in Korea from 2005 to 2014. 
J Korean Med Rehabil. 2015;25(2):89-96.

8. Jo HG, Park AR, Kee YB, Kang DH, Choi JB, Sul JU. A clinical trial on the blood stasis and efficacy of Kyejibokryongwhan(Guizhifuling-wan) in the patients with motor vehicle accident. J Korean Med Rehabil. 2011;21(3):45-55.

9. Korean Pharmacopuncture Institute Science Committee, Pharmacopuncturology Institute. Pharmacopuncturology. 2nd ed. Seoul: Elsevier Korea; 2011. p. 3-5, 9-17, 28-9, 45, 229-41.

10. Yun JM, Kim KH, Oh YT, Kim JU, Yook TH. The analysis of the recent research trend of pharmacopuncture. J Soc Prev Korean Med. 2018;22(2):55-63.

11. Lee JY, Han YJ, Kim JH, Kim YJ, Kwon KR. Type analysis of pharmacopuncture papers published in the Journal of Korean Institute of Pharmacopuncture. J Pharmacopuncture. 2006;9(3): 147-54.

12. Baik SI, Ahn JC, Kim YJ, Kim HS, Kwon KR. Type analysis of pharmacopuncture papers published in the Journal of Korean Acupuncture and Moxibustion. J Korean Acupunct Moxib Soc. 2006;23(6):19-27.

13. Kim YJ, Kim TR, Woo CH, Park JH. The retrospective analysis of traffic accident inpatients in Korean and Western medicine hospital. J Korean Med Rehabil. 2016;26(4):97-105.

14. Lee JH, Kim JS, Yang KY, Han SY, Lee CR, Shin HK. The comparative study on the effect of pharmacopuncture treatment and Chuna treatment for neck pain caused by traffic accidents. J Korean Med Rehabil. 2012;22(1):75-83.

15. Kim TH, Park WH, Cha YY. A retrospective study on the effects of ShinBaro pharmacopuncture and Jungsongouhyul pharmacopuncture on whiplash injury by traffic accident. J Korean Med Rehabil. 2013;23(4):1-7.

16. Woo JH, Han KW, Kim ES, Lee SJ, Lee JS, Nam JH, et al. A clinical study comparing the effects of Chuna treatment and Ouhyul herbal acupuncture on the patients with cervical pain caused by traffic accidents. J Korean Acupunct Moxib Soc. 2011;28(5):918.

17. Lee JS, Lee SJ, Nam JH, Kim KW, Lee MJ, Lim SJ, et al. The comparative study on the effect of pharmacopuncture treatment, Chuna treatment, pharmacopuncture-Chuna cooperative treatment for neck pain caused by traffic accidents. J Korean Acupunct Moxib Soc. 2012;29(5):87-95.

18. Song BY. A clinical study on the effects of sweet bee venom herbal acupuncture for patients with whiplash injury. J Pharmacopuncture. 2007;10(3):77-83.

19. Im JG, Lee JB, Lee HG, Yook TH, Kim JU. Effects of the acupuncture therapy in combination with Soyeom pharmacopuncture therapy on acute whiplash injury by traffic accident. J Korean Acupunct Moxib Soc. 2011;28(4):9-18.

20. Lee YC, Nam DJ, Huh G, Lee JE, Kim B, Song EM, et al. The comparative study on the effect of HWANGRYUNHAEDOKTANG pharmacopuncture and essential bee venom pharmacopuncture for cervical pain caused by traffic accidents: a randomized controlled trial. J Korean Med Rehabil. 2014;24(4):145-53.

21. Kim KW, Lee MJ, Jun JY, Lim SJ, Lee CH, Kim HS, et al. The comparitive study on the effect of Shinbaro pharmacopuncture and bee venom pharmacopuncture treatment of patient diagnosed as cervical disc hermiation based on the MRI after traffic accident: a retrospective, case series observational study. Acupuncture 2014;31(1):43-50.

22. Kim KH, Nam DW, Kang JW, Lee JD, Choi DY. Acupuncture for rehabilitation in patients with traffic accident in South Korea: a systematic review. J Korean Acupunct Moxib Soc. 2010;27(1):219.

23. Korean Pharmacopuncture Institute Science Committee, Pharmacopuncturology Institute. Pharmacopuncturology. 2nd ed. Seoul: Elsevier Korea; 2011. p. 13-62.

24. Textbook Compilation Committee for Korean Acupuncture Society. The acupuncture and moxibustion (Middle). Paju: Jipmoondang. 2008. p. 408.

25. Lim GM, Wi DY, Lee JH, Ko YS. The effects of Soyeom and Jungsongouhyul pharmacopuncture on whiplash injury by traffic accident. J Orient Rehabil Med. 2012;22(2):185-92.

26. Hwang DY. Bangyakhappyun. 2nd ed. Seoul: Yeonglim Publishing Inc; 2011. p. 264.

27. Korean Pharmacopuncture Institute Science Committee. Pharmacopuncturology. Seoul: Elsevier Korea; 2008. p. 3-8, 163-80, 259-420.

28. Lee SH, Kim SJ. An intensive review on clinical thesis about Hwangryunhaedok-tang pharmacopuncture treatment: focused on case reports and controlled studies in Korean Academic Journals. J Korean Med Rehabil. 2019;29(2):171-88.

29. Koh HK. Bee venom therapy. J Kyunghee Orient Med Coll. 1997;1:3-6.

30. Kim HJ, Ji YS, Lee SM, Jeon JH, Kim YI. A systematic review of clinical study of bee venom acupuncture. J Korean Acupunct Moxib Soc. 2013;30(4):151-9.

31. Kim SL, Hong KE. The clinical observations of 3 cases of metatarsal tendinitis treated with anti-inflammatory herbal acupuncture. J Pharmacopuncture. 2007;10(1):157-62.

32. Song HG, Choi JY, Kang JH, Lee H. The effect of the acupuncture therapy in combination with Soyeom pharmacopuncture therapy on the improvement of the symptoms of the patients with herniated intervertebral disk of L-spine in his initial stage of hospitalization. J Pharmacopuncture. 2009;12(4):111-8.

33. Jun BC, Kim ES, Kim DS, Kim TH, Kim JY. Effectiveness of ShinBaro pharmacopuncture on lumbar spinal herniated intervertebral disc: a randomized controlled trial. J Korea Chuna 
Jung Min Yun, et al.

Man Med Spine Nerves. 2011;6(2):109-19.

34. Ro HR, Park SH, Lee JY, Choo WJ, Han SW, Kim SW, et al. The comparative study on the effect of ShinBaro pharmacopuncture treatment and bee venom pharmacopuncture treatment of patient with spondylolisthesis. J Korea Chuna Man Med Spine Nerves. 2012;7(2):53-61.
35. Han KI, Jeon YT, Sin SH, Lee JH, Ko YS. The retrospective comparative study on the effect of muscle relaxation pharmacopuncture and Chuna manual therapy for neck pain caused by traffic accidents. J Korea Chuna Man Med Spine Nerves. 2016;11(1): 25-32. 